in 2017, St Giles Hospice in collaboration with Green Square Accord launched a four-week carers' course which was designed to support unpaid carers in the community who support people living with dementia. The course covers a range of topics, including looking after yourself and activities for people with dementia, eating and drinking and infections in dementia, dementia related behaviours and planning for the future including end-of-life.

Prior to 2020 this four-week course was face-to-face and was group based. When the COVID-19 pandemic hit the service had to act quickly to ensure the much-needed support for these carers did not disappear. The carers' course was adapted to provide support via a one-to-one basis either via video consultation or through a telephone call. This ensured that carers were not isolated and had access to specialist support and signposting to other services. Data captured showed that $100 \%$ of participants found that the topics covered were relevant to their situations, 92\% of participants found that sessions fulfilled their expectations and that $100 \%$ were given opportunity to ask questions. Over 80 carers have been supported through the adaptation of the service so far.

\section{P-117 LAST DAYS MATTER - A PROGRAMME TO HELP LAY FAMILIES LOOK AFTER THEIR LOVED ONES AT HOME}

Sophy Horner, Maddy Bass, Sue McGraw. St John's Hospice, Lancaster, Lancashire

\subsection{6/spcare-2021-Hospice.134}

Many people would like to die at home, but their loved ones can be nervous about looking after them fearing their own lack of clinical knowledge. Family and local health system engagement told us there was a gap in the provision of nonclinical family education.

'Last Days Matter' is the idea of the North Lancashire Compassionate Communities Group, who after research decided to develop their own programme that would support people without clinical expertise to look after their loved ones at home. The Group used their lay and clinical experiences to develop a three-hour programme consisting of five sessions. Each session is made up of a short film that follows the experience of John who is dying, and discussion time. Each session addresses a need that engagement had revealed; planning for the future; to care and accompany; saying goodbye, recognising the signs of dying and the new normal.

We learnt that it was necessary to seek funding for professional actors and filming skills so that we could have a programme that would be professional and how important it was to seek the expertise of the patient and public for their feedback on all scripts and teaching materials.

The result is a programme that is now ready to be used in local communities and has attracted the interest of North West NHS England and NHS Improvement. The programme will be made available further afield for adoption or adaption. It will support people to care for their loved ones at home by giving them the knowledge, reassurance and some of the necessary skills. Initial feedback from engagement and contributors suggests that 'Last Days Matter' is much needed, will be sought after and will be valued by those who need it most. It will also support palliative care staff across the health community.

\section{P-118 IMPROVING CARER SUPPORT IN HOSPICE CARE}

Amanda Wilkins, Barbara Ruff, Zoe Holman. St. Margaret's Hospice Care, Taunton and Yeovil, UK

\subsection{6/spcare-2021-Hospice. 135}

Background Carers provide vital support to patients yet manage a variety of emotional and physical demands themselves (Ates, Ebenau, Busa, et al., 2018). Use of 'carer needs' tools have been shown to reduce carer strain (Aoun, Grande, Howting, et al., 2015). Tools need to be part of wider organisational behavioural and cultural shift to improve person-centred support for carers (Diffin, Ewing, Harvey, et al., 2018). A quality improvement approach encompasses the wider factors involved in changing behaviours and culture in an organisation (NHS England. NHS Change Model).

Aims To evaluate the effectiveness of a quality improvement programme targeting carer support at the hospice.

Methods Baseline audit of 18 records carried out (2019) against the following standards; carers identified, relationship of carer documented, main carer identified, carer limitations documented, identification of needs, separate carer record and carer needs addressed.

Measures implemented share the vision, carer champions identified, record templates modified, staff awareness and process training, promotion of carer resources, embed change. Reaudit of 15 records (2021) to identify impact of improvements.

Results In only 72\% of cases a carer was identified in 2019 compared to $100 \%$ at review. Compliance with five of the standards in 2019 was between $17 \%$ and $22 \%$ of the records audited. Average compliance across seven standards in 2019 was 39\% and in 2021 compliance was 71\%. The re-audit identified a marked improvement in six of the standards audited. In over $90 \%$ of cases, the relationship of carer to patient was recorded successfully in both audits.

Conclusion Compliance with the standards increased by $32 \%$ on average. The measures implemented were effective at increasing the evidence and awareness of carer support in the organisation. Further improvements are needed in: where information is recorded, assessment of carer limitations and recording of intervention outcomes. Further quality improvements planned include sharing the outcome of the re-audit to provide ongoing momentum, increase number of carer champions and carer support to be incorporated at strategic level.

\section{P-119 SOLIDARITY, SKILLS AND SUPPORT- ST CATHERINE'S (LANCASHIRE) 'WINTER PRESSURES' CARE HOMES PROJECT}

${ }^{1,2}$ Claire Capewell, ${ }^{2}$ Deborah Bolton, ${ }^{2}$ Katherine Wilkinson, ${ }^{2}$ Victoria Hargreaves. ${ }^{1}$ Lancashire Teaching Hospitals NHS Trust, Preston, UK; ${ }^{2}$ St Catherine's Hospice, Preston, UK

10.1136/spcare-2021-Hospice. 136

Background Care homes have been particularly badly affected by the events of 2020 . We acquired short-term resource from two streams to improve system resilience during winter.

Aims To assess the impact of enhanced specialist palliative care to care homes regarding responsiveness, professional support and education, referrals, advance care planning (ACP), hospital admissions and deaths in preferred place of death (PPD).

Methods A free education programme was offered to all care homes in Preston, Chorley and South Ribble commencing in 